\title{
(2) OPEN ACCESS \\ Effect of syphilis infection on HIV acquisition: a systematic review and meta-analysis
}

\author{
Meng Yin Wu, ${ }^{1}$ Hui Zi Gong, ${ }^{1}$ Kui Ru Hu, ${ }^{2}$ He-yi Zheng, ${ }^{1}$ Xia Wan, ${ }^{2}$ Jun Li (1) ${ }^{1}$
}

Department of Dermatology and Venereology, Peking Union Medical College Hospital, Chinese Academy of Medical Sciences and Peking Union Medical College, Beijing, China 2Epidemiology and Statistics, Institute of Basic Medical Sciences at Chinese Academy of Medical Sciences \& School of Basic Medicine at Peking Union Medical College, Beijing, China

Correspondence to Dr Jun Li, Department of Dermatology and Venereology, Peking Union Medical College Hospital, Chinese Academy of Medical Sciences and Peking Union Medical College, Beijing, China; lijun35@hotmail.com

$\mathrm{XW}$ and $\mathrm{JL}$ are joint senior authors.

Received 15 July 2020

Revised 12 October 2020

Accepted 25 October 2020

\begin{abstract}
Objectives Co-infection of syphilis and HIV remains hard to manage and its morbidity shows a rising tendency. Syphilis has been associated with increased risk of HIV acquisition in high-risk groups, especially in men who have sex with men (MSM). This systematic review and meta-analysis estimates the effect of syphilis infection on subsequent HIV acquisition, and assesses its difference between MSM and other high-risk populations
\end{abstract}

Methods Five electronic databases were searched for literature published to 21 September 2019 without language restrictions. Longitudinal studies that enrolled key populations to compare the incidence of HIV with and without syphilis exposure were included. We used a random-effects model to estimate the effect of syphilis infection on HIV acquisition among high-risk populations, which include MSM, sex workers, serodiscordant couples, people who inject drugs and attendees of STD clinics. Results A total of 17 cohorts and 5 case-control studies involving 65232 participants were included. HIV incidence showed a two-time increase after syphilis exposure, compared with a control group (relative risk (RR) 2.67 (95\% Cl 2.05 to 3.47); $p<0.05$ for prevalence; RR 3.21 ( $95 \%$ Cl 2.26 to 4.57); $p=0.419$ for incidence). No significant differences were observed between MSM and other high-risk groups in syphilis infection prevalence (RR 2.60 (95\% Cl 1.78 to 3.80); $p<0.05$ vs $R R, 2.98$ ( $95 \% \mathrm{Cl} 2.15$ to 4.14$) ; p<0.05$; ratio of relative risk $0.76(95 \% \mathrm{Cl} 0.49$ to 1.17$))$.

Conclusions Syphilis infection increases the risk of HIV acquisition in high-risk populations. There is no evidence to suggest MSM are at greater risk than other highrisk populations. Prompt diagnosis, timely treatment, preventive interventions against syphilis infection would be a worthwhile investment for reducing HIV incidence. Strategies to combat stigma and discrimination targeted at MSM are pragmatically needed.

\section{INTRODUCTION}

By the turn of the century, syphilis had resurged as a global public health burden with a high prevalence. It was estimated that there were 6 million new infections of syphilis worldwide in $2016,{ }^{1}$ primarily affecting men who have sex with men (MSM) and often accompanied by HIV co-infection. Over the past decade, the trend of rising syphilis rates among MSM has continued unabated. From 2000 to 2013, the proportion of reported syphilis cases in highincome countries that occurred in MSM increased from $26.8 \%$ to $55 \%$, and more than half of these countries recorded an increased prevalence in the MSM group. ${ }^{2}$ Although syphilis surveillance is limited in low-income and middle-income countries, these locations generally have higher syphilis burdens. According to the newest report published by WHO (2018), the median syphilis seroprevalence was 6.0\% (range: 0\%-36.7\%) among the MSM group, with the highest seroprevalence values being $>20 \%$ in five countries (Brazil, Colombia, Fiji, Georgia and Mexico) (2016-2017). ${ }^{1}$

Syphilis and HIV are two systemic, STDs that share common risk factors. Previous research suggested that the severe complications that are attributed to untreated syphilis can facilitate the transmission and acquisition of HIV infection. In turn, co-infection with HIV could alter the syphilis manifestations and blur distinction of the stages, potentially misleading clinical judgement. HIV/ syphilis co-infection patients may also face a higher risk of treatment failure, and their genital ulcers generally take longer to heal than those of patients with syphilis alone, which may increase the risk of exposure to other STDs. ${ }^{3}$ In the MSM group, syphilis surveillance showed an increasing HIV and syphilis co-infection rate, ranging from $30 \%$ to $60 \%$ depending on the geographic location. ${ }^{13-6}$ Due to the reciprocal synergistic interaction between syphilis and HIV, the rising epidemic of this concomitant HIV infection among MSM remains hard to manage. Given the potential effects of syphilis prevention on reducing the HIV incidence, quantifying the association between these two pathogens has great public health implications.

Three meta-analytic systematic reviews studying the risk factors of HIV infection in their own country have summarised the association between syphilis and HIV: two of them focused on the MSM group in China (one in $2011^{7}$ and the other in 2015), ${ }^{8}$ and the one in India analysed the situation for each gender (published in 2012). ${ }^{9}$ These articles reveal a twofold to threefold increase in the risk of HIV infection with baseline syphilis infection. However, the causal relationship was not clear due to the inclusion of cross-sectional studies. Here, we use a wider range of publication sources (worldwide rather than country-specific) to update and re-quantify the effect of syphilis infection on HIV acquisition based on high-quality evidence, with the goal of better elaborating the benefits of syphilis infection prevention efforts on HIV reduction. Estimates of syphilis infection prevalence and incidence are provided separately for MSM and other high-risk populations. 


\section{METHODS}

\section{Search strategy}

This systematic review and meta-analysis was performed in accordance with the Preferred Reporting Items for Systematic Reviews and Meta-Analyses (http://www.prisma-statement.org/) and Meta-analysis of Observational Studies in Epidemiology guidelines. ${ }^{10}$ For our search strategy, we searched PubMed, Embase, MEDLINE, Web of Science and Cochrane Library without language restrictions to identify all relevant studies on HIV acquisition after exposure to syphilis infection, published to 21 September 2019. For PubMed, we searched for articles and abstracts using the terms ('syphilis', 'lues', 'Treponema pallidum' OR 'T. pallidum') AND ('HIV', 'AIDS', 'human immunodeficiency virus', 'human immuno deficiency virus', 'human immunedeficiency virus', OR 'human immune deficiency virus'). This presented strategy was adapted to fit with each of the other databases. A further screening of the references cited in all relevant studies was performed to identify potential data sources. We also contacted authors regarding unpublished data and unclear data.

\section{Study selection}

Studies were included in our systematic review (for full details of the selection criteria, see online supplemental table 1) if they were a cohort study, controlled trial or case-control study (including nested case-control study) comparing the effect of preceding syphilis infection on HIV acquisition with a syphilisnegative group. An unexposed group was defined as syphilisnegative if they remained negative for syphilis throughout the follow-up or at baseline (for studies in which a syphilis retest was not done during follow-up). We excluded cross-sectional studies, studies with no primary data, studies relying on self-reported syphilis infection history and studies limited to syphilis infected and/or HIV-infected individuals only (ie, no uninfected participants were included).

Two authors (MYW and JL), independently and in duplicate, screened the title, abstracts and tables of articles retrieved from the literature search, and full texts of potentially eligible articles were downloaded and further assessed based on the inclusion criteria. Disagreements were resolved through discussions between these two authors until a consensus was reached.

\section{Data extraction}

Two reviewers (MYW and JL) independently extracted data and assessed study quality following the Newcastle-Ottawa Scale $(\mathrm{NOS})^{11}$ guidelines (see online supplemental tables 2 and 3). When a database appeared to be inconsistent, the controversial article would be re-evaluated and fully discussed between the two authors until a consensus was reached. Publications reporting on the same region but during different periods were considered as a single trial.

Data extracted included participant characteristics (mean or median age, population, world region, baseline syphilis prevalence and HIV acquisition rate), study characteristics (first author, publication year, study design, syphilis confirm test, follow-up rate, length of time between tests, definition of unexposed group and timing of incident syphilis infection relative to HIV acquisition) and adjustment of confounding factors (condom use, male circumcision status, genital ulcer diseases, any sexual behaviour, number of sexual partners, age and drug use).

To minimise biases due to reverse causation, we classified timing sequences into three subcategories: (1) syphilis seroconversion was verified to occur in a previous time interval before
HIV acquisition (definitely before); (2) syphilis seroconversion happened in a previous or in the same interval as HIV acquisition ('before and indeterminably close') and (3) syphilis might have happened after HIV infection ('after and indeterminably close').

\section{Quality assessment}

We assessed the quality of individual trials using the NOS to evaluate five key domains: selection of study participants, sample representativeness (epidemiological core groups), ascertainment of exposure or outcome, confounding of the study design or analysis and other sources of bias. One star would be awarded for each item if the study met the defined criteria.

\section{Data analysis}

We used the meta packages of STATA (V.15.0) to perform the analysis. Data were combined using a random-effects model based on the DerSimonian-Laird inverse-variance method ${ }^{12}$ to pool the weighted relative risk (RR) of HIV acquisition risk estimates. Ratio of relative risk (RRR) and its CI were calculated as an estimated interaction effect for comparing $\mathrm{RR}^{13}$ (http://www. hutchon.net/CompareRR.htm). Heterogeneity across studies was assessed by using $\chi^{2}$ (threshold $p=0.10$; a $p$ value of $<0.10$ indicates statistically significant publication bias) and quantified by the $\mathrm{I}^{2}$ statistic, for which a value of $50 \%$ was considered to be acceptable. We explored potential sources of heterogeneity with subgroup analyses for each of the study-level characteristics. Publication bias was assessed by using the symmetry of funnel plots and Egger's test at estimate level. Sensitivity analysis was conducted to detect potential outliers.

\section{RESULTS}

\section{Study selection}

Our search strategy identified 2821 publications (figure 1). Of these, 130 were rejected due to duplication, and 2691 publications remained for screening. After screening, 207 articles were assessed for eligibility. Finally, we identified 22 qualified publications ${ }^{14-35}$ from 19 independent studies on the basis of our selection criteria. The results of the NOS assessment for risk of bias within studies are summarised in online supplemental table 4. A six-star or seven-star rating was the most common with 17 studies (77\%). Seventeen studies reported primary data based on prevalent syphilis infection, and five studies reported the events of HIV acquisition after exposure to incident syphilis infection. Sixteen studies focused on MSM, and six studies reported on other high-risk groups, including sex workers, serodiscordant couples, people who inject drugs and attendees of STD clinics. Eleven of studies (50\%) were conducted in Asia, in populations where baseline syphilis prevalence ranges from $10 \%$ to $30 \%$.

\section{Study characteristics}

Table 1 summarises the characteristics of included studies and participants. The 22 included studies enrolled a total of 65232 participants from five continents. Eleven of the studies (50\%) were from Asia (China, China-Hong Kong and Thailand), three studies (13.6\%) were from Europe (UK, Spain and Germany), three studies (3.6\%) were from the Americas (the USA), three studies (13.6\%) were from Africa (Tanzania, Rwanda and Uganda) and two studies (9.1\%) were from Oceania (Australia). Eighteen studies $(81.8 \%)$ were cohort studies, and four studies $(8.2 \%)$ were case-control studies. The mean or median age of participants varied from 24 to 36. 
2821 records identified through database searching

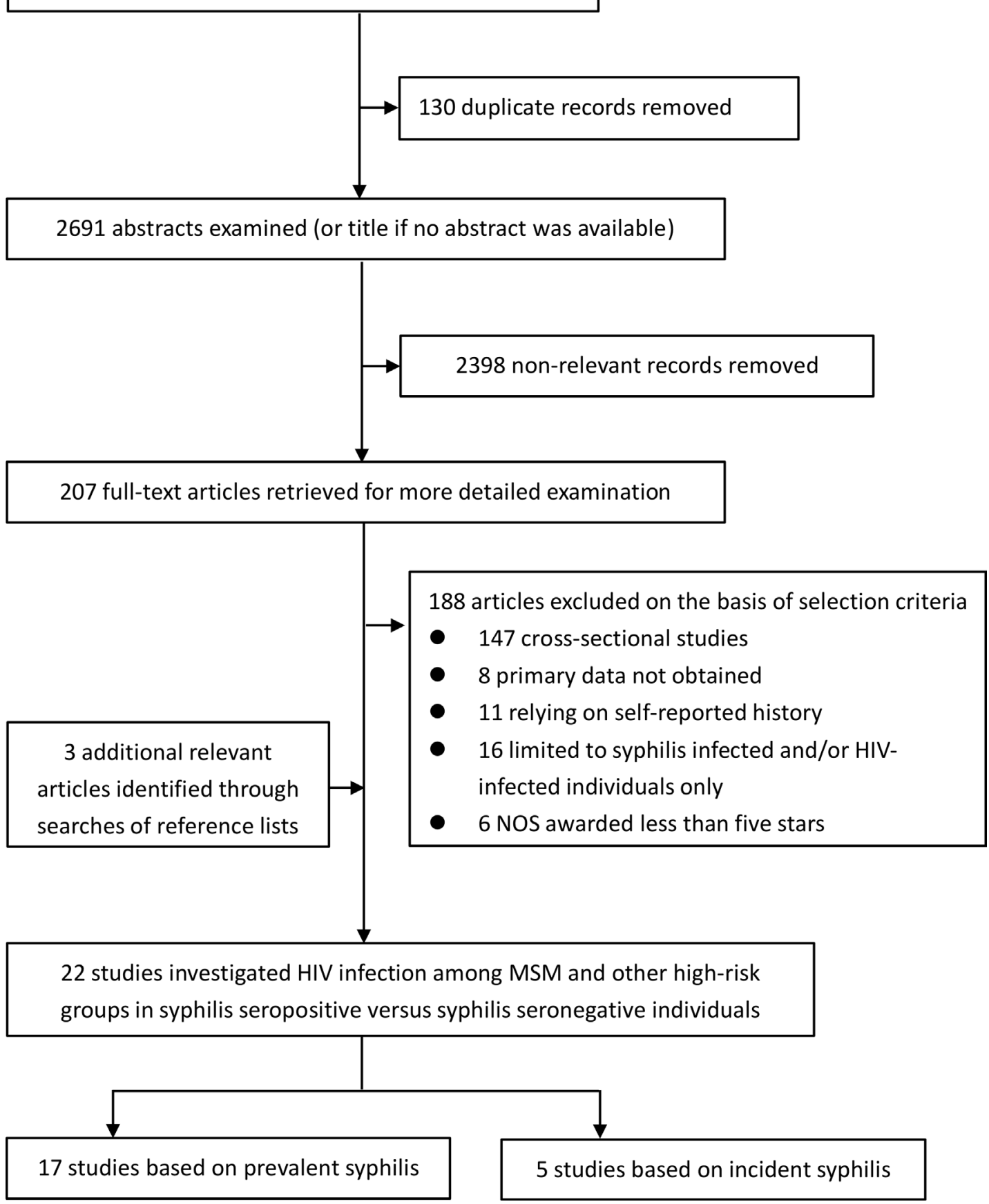

Figure 1 Flow diagram for selection of studies included in the systematic review and meta-analysis. MSM, men who have sex with men; NOS, Newcastle-Ottawa Scale (for assessing the quality of non-randomised studies in meta-analyses).

Twenty (90.9\%) of the studies tested for Treponema pallidum using at least one decentralised method at the point of care (17 studies tested with non-treponemal tests (NTTs) and confirmed with treponemal tests (TTs), 3 studies only tested with NTTs); the other two studies (9.1\%) did not report their specific test method. Twelve (54.5\%) studies re-tested at least every 6 months, whereas seven studies $(31.8 \%)$ performed re-tests at intervals of $>6$ months. The unexposed group was defined as syphilis-negative throughout follow-up in 10 of the studies $(45.5 \%)$, and 7 studies (31.8\%) set syphilis-negative at baseline.

\section{Associations between syphilis infection and HIV acquisition}

According to the random-effects meta-analysis (figure 2), the incidence of HIV was significantly higher among patients with syphilis infection than in the corresponding syphilis-negative control group, especially in the incident syphilis infection group (RR 3.21, 95\% CI 2.26 to $4.57 ; \mathrm{n}=5, \mathrm{I}^{2}=0 \% ; \mathrm{p}=0.419$ ). For exposure to prevalent syphilis infection, the pooled RR was 2.67 (95\% CI 2.05 to $\left.3.47 ; \mathrm{n}=17, \mathrm{I}^{2}=45.5 \% ; \mathrm{p}<0.05\right)$. The results and the magnitude of the associations for other high-risk populations were similar to those for MSM populations (RR 2.98, 95\% CI 2.15 to $4.14 ; \mathrm{n}=5 ; \mathrm{p}=0.861$ vs RR $2.60,95 \%$ CI 1.78 


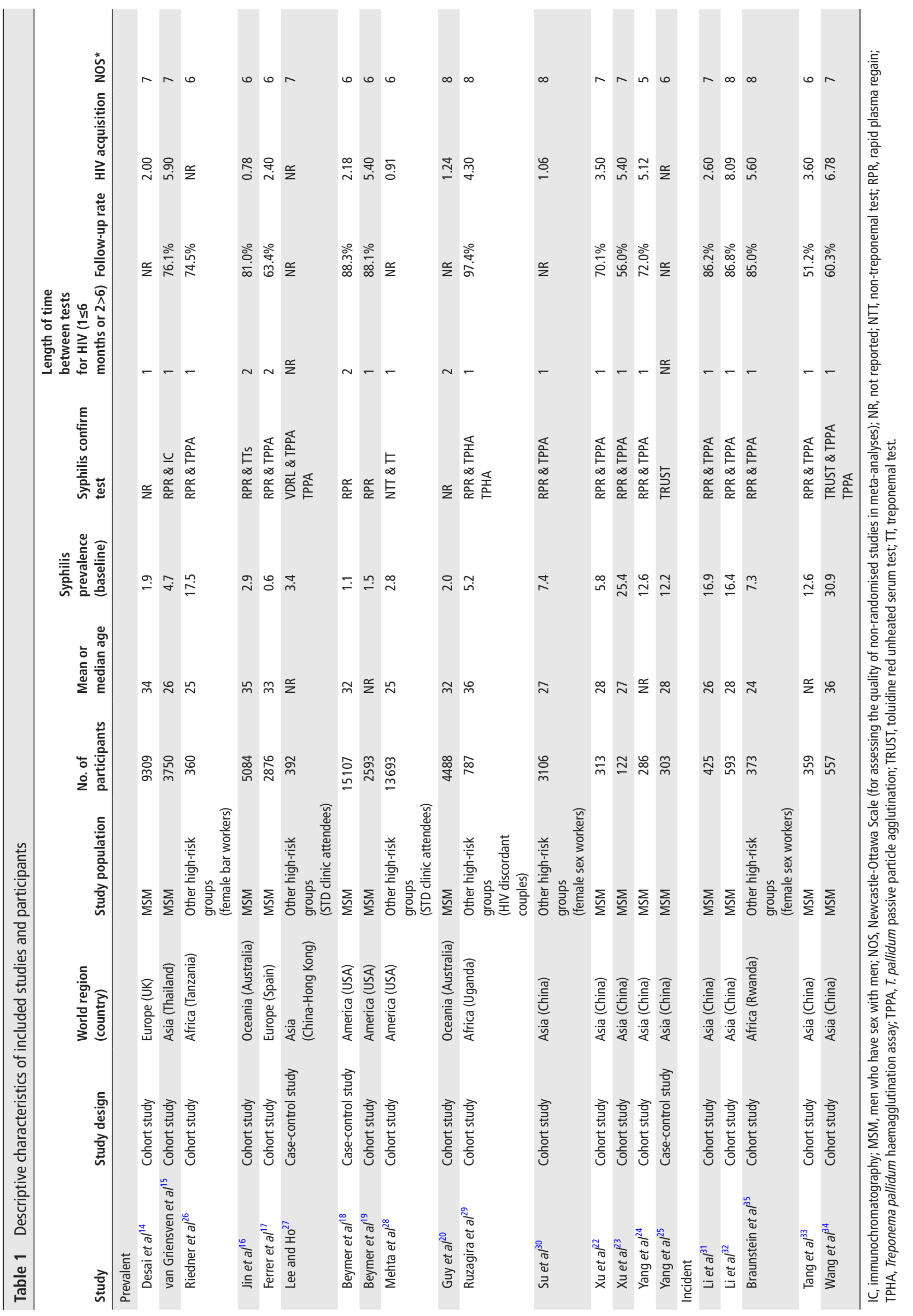




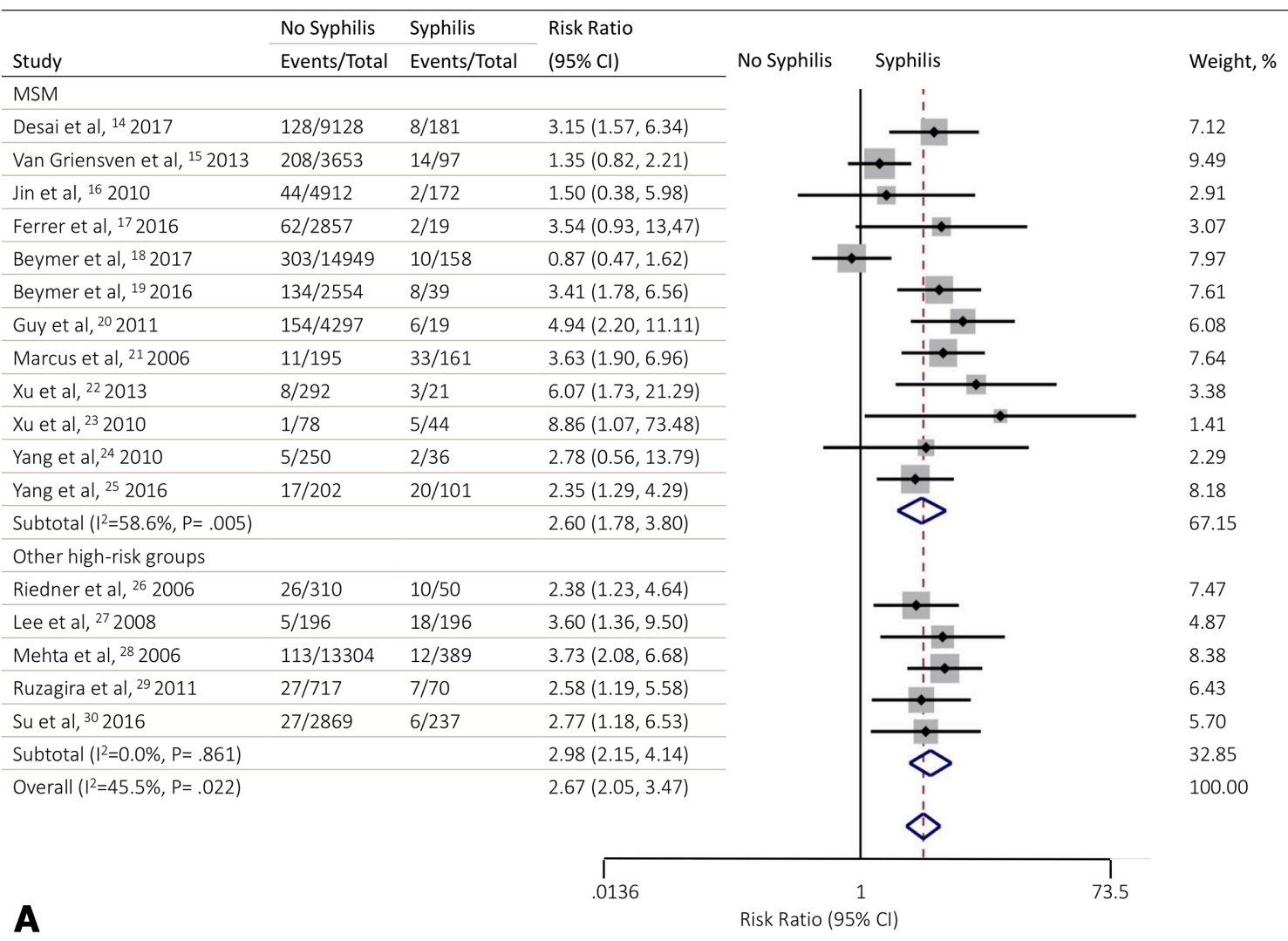

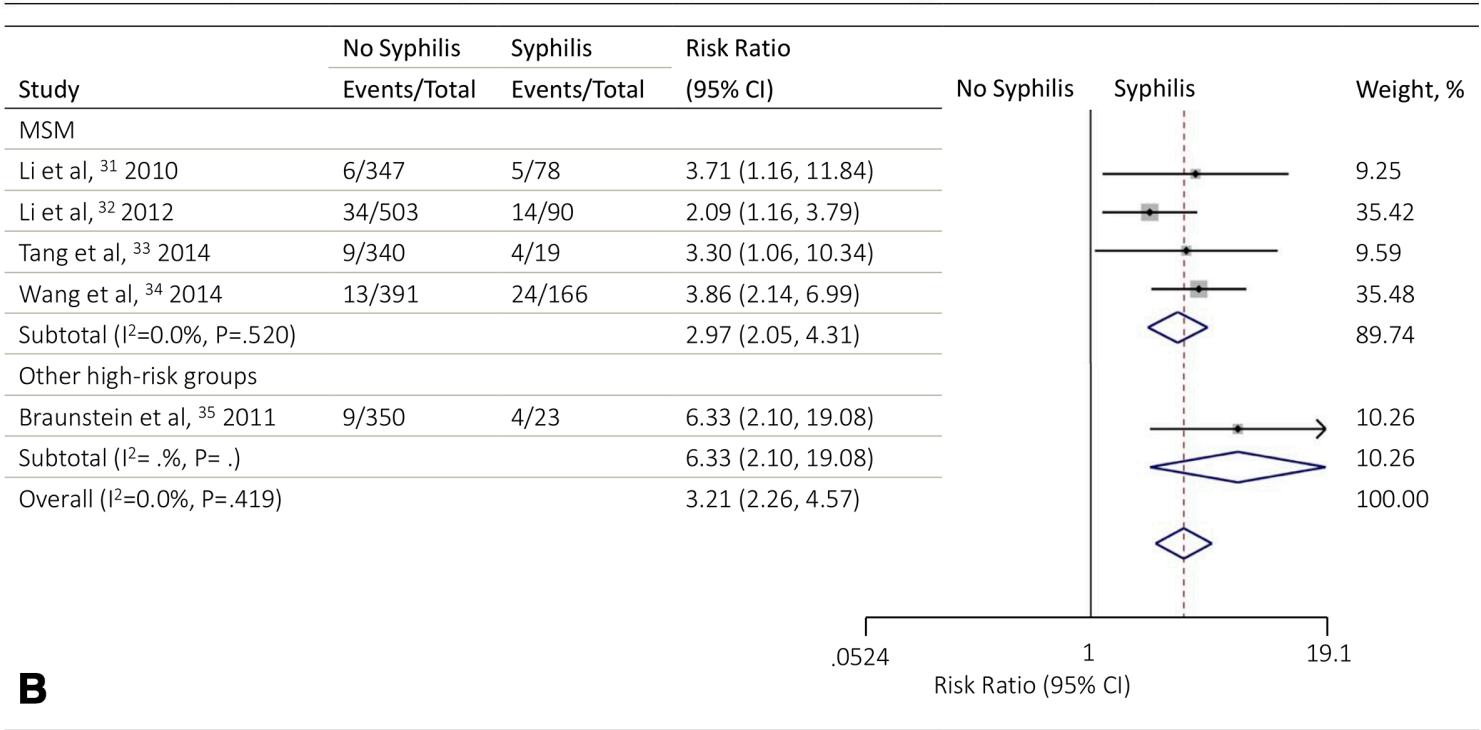

Figure 2 Forest plots. RR estimates of the association between exposure to syphilis infection and HIV incidence. (A) Prevalent syphilis infection and (B) Incident syphilis infection. MSM, men who have sex with men; RR, relative risk. Note: estimates for MSM and other high-risk populations are shown separately. Estimates for other high-risk populations (ie, sex workers, serodiscordant couples, people who inject drugs and attendees of STD clinics) were combined because they could not be separated by gender.

to $3.80 ; \mathrm{n}=12 ; \mathrm{p}<0.05$, respectively) in the prevalent group. Transforming into the RRR scale, the comparison of two estimated RR showed no good evidence to support a different outcome between MSM and other high-risk populations (RRR 0.76 (95\% CI 0.49 to 1.17$)$ ). For exposure to incident syphilis infection, the comparison of two populations was not statistically significant due to the limited study on other high-risk groups (only one study in comparator group, Braunstein et $a l^{35}$ ). Consequently, the conclusion needs to be further verified with more high-quality clinical researches.
In a subgroup analysis (table 2, see online supplemental figures 1-10), no significant differences were seen in the syphilis/HIV associations by age, baseline syphilis prevalence, syphilis confirm test, length of time between tests, follow-up rate or timing sequences. However, it should be noted that the subgroup analysis for incident syphilis infection by age and baseline syphilis prevalence has no statistical differences for its lack of comparator group. Estimates of the syphilis/HIV association tended to be lower for Australia (RR 2.08, 95\% CI 1.02 to 4.26; $\left.\mathrm{n}=2, \mathrm{I}^{2}=0.0 \% ; \mathrm{p}=0.439\right)$ than for other regions, which held a 
Table 2 Description of studies and RR estimates of the association between syphilis infection and HIV incidence by characteristics and participants characteristics

\begin{tabular}{|c|c|c|c|c|}
\hline & No. of studies & RR $(95 \% \mathrm{CI})$ & Variance explained $\mathrm{I}^{2}$ & $P$ value \\
\hline \multicolumn{5}{|l|}{ Participant characteristics } \\
\hline \multicolumn{5}{|l|}{ Mean or median age } \\
\hline$\leq 30$ years $*$ & 7 & 2.53 (1.50 to 4.26$)$ & $64.3 \%$ & 0.010 \\
\hline$t$ & 3 & $3.20(1.62$ to 6.30$)$ & $39.4 \%$ & 0.192 \\
\hline$>30$ years* & 7 & 2.60 (1.76 to 3.84$)$ & $46.1 \%$ & 0.084 \\
\hline$\dagger$ & 1 & 3.86 (2.14 to 6.99$)$ & - & - \\
\hline \multicolumn{5}{|l|}{ Population } \\
\hline $\mathrm{MSM}^{*}$ & 12 & 2.60 (1.78 to 3.80$)$ & $58.6 \%$ & 0.005 \\
\hline$\dagger$ & 4 & 2.97 (2.05 to 4.31$)$ & $0.0 \%$ & 0.520 \\
\hline Other high-risk groups* & 5 & 2.98 (2.15 to 4.14$)$ & $0.0 \%$ & 0.861 \\
\hline$t$ & 1 & 6.33 (2.10 to 19.08$)$ & - & - \\
\hline \multicolumn{5}{|l|}{ World region* } \\
\hline Europe & 3 & $3.26(2.08$ to 5.11$)$ & $0.0 \%$ & 0.880 \\
\hline Asia & 11 & 2.80 (2.20 to 3.56$)$ & $0.0 \%$ & 0.710 \\
\hline Africa & 3 & 2.77 (1.65 to 4.66$)$ & $16.6 \%$ & 0.301 \\
\hline Australia & 2 & 2.08 (1.02 to 4.26$)$ & $0.0 \%$ & 0.439 \\
\hline America & 3 & 3.32 (2.32 to 4.73$)$ & $0.0 \%$ & 0.920 \\
\hline \multicolumn{5}{|l|}{ Syphilis prevalence (baseline) } \\
\hline$\leq 10 \% *$ & 12 & 2.63 (1.84 to 3.77$)$ & $59.7 \%$ & 0.004 \\
\hline$t$ & 1 & 6.33 (2.10 to 4.57$)$ & & \\
\hline$>10 \%$ * & 5 & 2.81 (1.97 to 4.00$)$ & $45.5 \%$ & 0.022 \\
\hline$t$ & 4 & 2.97 (2.05 to 4.31$)$ & $0.0 \%$ & 0.520 \\
\hline \multicolumn{5}{|l|}{ Study characteristics } \\
\hline \multicolumn{5}{|l|}{ Study design } \\
\hline Cohort study* & 13 & 2.83 (2.18 to 3.69$)$ & $23.1 \%$ & 0.210 \\
\hline$\dagger$ & 5 & $3.21(2.26$ to 4.57$)$ & $0.0 \%$ & 0.419 \\
\hline Case-control study* & 4 & 2.20 (1.11 to 4.37$)$ & $74.5 \%$ & 0.008 \\
\hline \multicolumn{5}{|l|}{ Follow-up rate } \\
\hline$\leq 80 \% *$ & 7 & 2.75 (1.71 to 4.41$)$ & $44.2 \%$ & 0.096 \\
\hline$\dagger$ & 2 & 3.74 (2.26 to 6.32$)$ & $0.0 \%$ & 0.419 \\
\hline$>80 \% *$ & 4 & 1.87 (0.88 to 3.97$)$ & $54.0 \%$ & 0.012 \\
\hline$\dagger$ & 3 & $3.20(1.62$ to 6.30$)$ & $39.4 \%$ & 0.192 \\
\hline \multicolumn{5}{|l|}{ Syphilis confirm test } \\
\hline NTT \& TT & 17 & 2.97 (2.43 to 3.62$)$ & $0.0 \%$ & 0.818 \\
\hline NTT & 3 & 2.77 (1.93 to 3.96$)$ & $0.0 \%$ & 0.534 \\
\hline \multicolumn{5}{|l|}{ Length of time between tests } \\
\hline$\leq 6$ months* & 10 & $2.74(2.04$ to 3.66$)$ & $27.7 \%$ & 0.189 \\
\hline$\dagger$ & 2 & 2.31 (1.36 to 4.57$)$ & $0.0 \%$ & 0.487 \\
\hline$>6$ months* & 4 & 2.61 (1.88 to 3.62$)$ & $53.8 \%$ & 0.009 \\
\hline$t$ & 3 & 4.21 (2.61 to 6.77$)$ & $0.0 \%$ & 0.715 \\
\hline \multicolumn{5}{|l|}{ Definition of unexposed group* } \\
\hline Syphilis (-) throughout follow-up & 10 & 3.31 (2.59 to 4.22$)$ & $0.0 \%$ & 0.858 \\
\hline Syphilis (-) at baseline & 7 & $1.97(1.25$ to 3.11$)$ & $45.5 \%$ & 0.022 \\
\hline \multicolumn{5}{|l|}{ Timing sequence } \\
\hline Definitely before & 13 & $2.83(2.28$ to 3.51$)$ & $0 \%$ & 0.840 \\
\hline Before and indeterminably close & 9 & 3.01 (2.33 to 3.90$)$ & $0 \%$ & 0.761 \\
\hline
\end{tabular}

*Prevalent syphilis infection.

tIncident syphilis infection.

MSM, men who have sex with men; NTT, non-treponemal test; RR, relative risk; TT, treponemal test.

similar pooled RR (95\% CI 2.77 to 3.32 ) and association magnitude. For exposure to prevalent syphilis infection, the risk of HIV acquisition was significantly higher in cohort studies (RR $2.83,95 \%$ CI 2.18 to $\left.3.69 ; n=13, I^{2}=23.1 \% ; p=0.210\right)$ than in case-control studies (RR 2.20, 95\% CI 1.11 to $4.37 ; n=4$, $\left.\mathrm{I}^{2}=74.5 \% ; \mathrm{p}<0.01\right)$; however, no study estimates were available for incident syphilis infection because lack of comparator group. The risk of HIV infection was statistically different by the definition used for the unexposed group; studies that defined the unexposed group as syphilis-negative throughout follow-up presented a higher RR (RR 3.31, 95\% CI 2.59 to 4.22 ; $n=10$, $\left.\mathrm{I}^{2}=0.0 \% ; \mathrm{p}=0.858\right)$ compared with those that defined this group as syphilis-negative at baseline (RR 1.97, 95\% CI 1.25 to $\left.3.11 ; \mathrm{n}=7, \mathrm{I}^{2}=45.5 \% ; \mathrm{p}<0.05\right)$. 
Publication bias was not significant according to either funnel plots or Egger's test (see online supplemental figures 11-16). Sensitivity analyses did not change the overall findings significantly (online supplemental figures 17; 18). All included studies were used for the meta-analysis.

\section{DISCUSSION}

This systematic review and meta-analysis provides high-quality evidence on the effect of syphilis infection on HIV acquisition. We minimised the risk of bias from different levels by strictly following inclusion criteria. Furthermore, the precise study information we extracted supported our ability to review the effects of the potential factors related to participant and study characteristics, allowing us to comprehensively assess the associations between syphilis and HIV infection. However, potential confounding factors from the original data were hard to control at the same level, so these could not be eliminated through our analysis.

Our results provide evidence that syphilis infection almost tripled the risk of HIV acquisition, which is in line with the results of most studies. ${ }^{28} 36$ Researchers believe this interaction is multifactorial. From a clinical perspective, syphilitic ulcers in the genital area may account for the higher rate of HIV virus transmission and acquisition. The impairment of the protective epithelial barrier by these ulcers provides a portal of entry to the causative agent of HIV, thus increasing the likelihood of infection. In addition, the lesion site is rich in macrophages and activated lymphocytes, ${ }^{37}$ which acts as an immuno-microenvironment of highly expressed receptors for HIV within the ulcer area and can facilitate the process of HIV acquisition. In the primary stage, regulatory T-cells are predominately responsible for the clearance of T. pallidum at local sites of infection, and they accomplish this by activating interferon- $\gamma$, which can help enhance the internalisation and degradation capacity of macrophages. ${ }^{38}$ However, activated macrophages have been shown to be associated with HIV co-infection for its resistance to the antiviral effects of chemokines. Numerous CD4-bearing macrophages and $\mathrm{T}$ lymphocytes that are attracted by chemokines will aggregate at the site of $T$. pallidum replication and thus, providing abundant target cells for HIV virus. ${ }^{39}{ }^{40}$ The inflammatory response to $T$. pallidum replication is complex and variable, and opsonic antibodies play a major role in promoting the killing and degradation of spirochetes within phagolysosomes. ${ }^{41}$ However, a small quantity of pathogens will effectively evade host opsonophagocytosis, thereby facilitating the transition into early latent syphilis or second syphilis. ${ }^{42}$ Consequently, T. pallidum has a reputation for immune-evasion stemming from its antigenic variation of TprK, an impaired antibody-mediated opsonisation, and a paucity of surface-exposed pathogen-associated molecular patterns. $^{43}$

On the molecular biology level, lipoproteins under the outer membrane of T. pallidum show strong immunogenicity and have been proposed as the major proinflammatory agonists of syphilis, ${ }^{44}$ activating macrophages and dendritic cells via the toll-like receptor 2 signal pathway. ${ }^{39}$ Lipoproteins were also found to be capable of inducing HIV-1 gene expression in human monocytic cells via a nuclear factor-kappa B-dependent mechanism, ${ }^{45}$ which may explain the transmission of the latent HIV virus in cases with syphilis infection. Moreover, lipoproteins could upregulate the expression of CCR5, the $\beta$-chemokine receptor, in human monocytes, ${ }^{44}$ thereby enhancing susceptibility to HIV infection. CCR5 is a principal coreceptor for HIV entry used by macrophage-tropic (M-tropic) strains, the most infectious and prevalent of HIV strains, and it was observed to be overexpressed on dendritic cells and $\mathrm{CD}^{+}{ }^{+}$cells. ${ }^{46} 47$ Additionally, syphilis infection has been indicated to enhance HIV replication by altering cell cycles via affecting cytokine secretion and upregulating the expression of transcription factors, especially during the second syphilis stage. ${ }^{48}$

Presently, exploration of the pathogenesis of syphilis and host immunity against it is restricted by the failed attempts to culture T. pallidum (ex vivo) and the limited choice of animal models, which hampers the development of effective prevention for syphilis. This underlines the importance of effective behavioural interventions and broad syphilis screening offers for key populations, which will also be critical in breaking the chain of HIV transmission.

Our findings have significant implications for the prevention of syphilis infection and management of HIV. In clinical work, knowledge of this association could prompt clinicians to advise individuals diagnosed with syphilis to do further HIV screening in case of a missed diagnosis and to educate these patients of their increased risk of acquiring HIV. Additionally, our findings support recommendations of the WHO information note published in $2017,{ }^{49}$ which aimed to generalise the implementation of dual HIV and syphilis point-of-care tests in highrisk populations. Although our subgroup analysis on interval between testing showed no statistical significance because of limited studies, we can still see a trend that more frequent testing resulting in less HIV burden. And there has been a focus on strategies to increase frequency of screening for HIV and STI testing as part of HIV monitoring in high-risk populations, which could promote early detection of asymptomatic syphilis and prevent secondary transmissions. At the prevention level, our results may promote policy makers and stakeholders to increase investment in the development of a syphilis vaccine, given the potential benefit it would have on reducing the HIV incidence. More notably, our results show that MSM are not at a higher risk when compared with other key populations, indicating the necessity of developing strategies to combat stigma and discrimination and to reduce violence targeted at MSM, as these are urgently required for alleviating the STD epidemic.

One potential source of heterogeneity in our analysis was the large variations in baseline syphilis prevalence in Asia, which were much higher than the average levels in other regions. The limitation in acquiring healthcare ${ }^{50}$ and the high false-positive rate of NTTs in syphilis screening may account for this inconsistency, resulting in a detection bias. Another confounding factor was misclassification bias regarding the exposure to syphilis infection. Misclassification bias can exist if syphilis exposure is defined solely according to baseline infection status, which could reduce the credibility of the result once some unexposed participants seroconvert to a syphilis-positive status during the study follow-up period. Even if those seroconversion cases were counted, the timing sequence between HIV infection and syphilis infection would be difficult to determine for the newly co-infected individuals, which might lead to another potential threat to validity. To minimise the risk of reverse causation, we excluded cross-sectional studies and performed a subgroup analysis based on timing sequence. The results and the magnitude of the associations did not show any significant differences.

Evidence derived from our systematic literature review supports the finding that syphilis infection can significantly increase the risk of HIV acquisition, in both MSM and other high-risk groups. Performing screening for key populations, enacting timely interventions against syphilis infection, and providing a proportional increase in research funding for vaccine 
development will be necessary for both syphilis prevention and a reduction in HIV acquisition.

\section{Key messages}

- Syphilis shares common risk factors with HIV and has been indicated to play a promotion role in HIV acquisition and sexual transmission.

- Currently, there are only country-specific evidence-based studies have summarised the association between syphilis and HIV, and the causal relationship was not definitive because of the inclusion of cross-sectional studies.

- This systematic review demonstrated an increased risk of HIV acquisition after syphilis exposure and found no significant difference in odds to be infected between MSM and other high-risk populations.

- Knowledge of this association is crucial in promoting the preventive interventions against syphilis in consideration of its potential benefits on HIV reduction; also, efforts to combat discrimination targeted at MSM group are urgently needed.

\section{Handling editor Laith J Abu-Raddad}

Contributors MYW and JL had full access to all of the data in the study and takes responsibility for the integrity of the data. MYW and XW participated in study concept and design, interpreted results and supervision. All authors participated indata collect. MYW, XW and KRH did the statistical analysis. MYW and JL wrote the draft report. All authors revised the report and approved the final version before submission.

Funding This work is supported by Beijing Municipal Science and Technology Commission (No. Z191100006619011) and the Capital's Funds for Health Improvement and Research (2020-2-4016).

Competing interests None declared.

Patient consent for publication Not required.

Ethics approval Ethics committee number: S-k653

Provenance and peer review Not commissioned; externally peer reviewed.

Data availability statement Data are available on reasonable request. The data from participants were extracted from previous publications. All data relevant to this study are included in the article and supplementary information. Also, data are available from the first author or corresponding author (mengyin.wu@foxmail.com, lijun35@hotmail.com).

Open access This is an open access article distributed in accordance with the Creative Commons Attribution Non Commercial (CC BY-NC 4.0) license, which permits others to distribute, remix, adapt, build upon this work non-commercially, and license their derivative works on different terms, provided the original work is properly cited, appropriate credit is given, any changes made indicated, and the use is non-commercial. See: http://creativecommons.org/licenses/by-nc/4.0/.

\section{ORCID iD}

Jun Li http://orcid.org/0000-0003-4820-5251

\section{REFERENCES}

1 World Health Organization. Report on global sexually transmitted infection surveillance 2018. Geneva:WHO, 2018.

2 Read P, Fairley CK, Chow EPF. Increasing trends of syphilis among men who have sex with men in high income countries. Sex Health 2015;12:155-63.

3 Centers for Disease Control and Prevention. Syphilis surveillance supplement 2013-2017. Atlanta, US: Department of Health and Human Services, 2019.

4 Zetola NM, Klausner JD. Syphilis and HIV infection: an update. Clin Infect Dis 2007;44:1222-8.

5 Jansen K, Schmidt AJ, Drewes J, et al. Increased incidence of syphilis in men who have sex with men and risk management strategies, Germany, 2015. Euro Surveill. 2016;21:30382

6 Wu Z, Xu J, Liu E, et al. Hiv and syphilis prevalence among men who have sex with men: a cross-sectional survey of 61 cities in China. Clin Infect Dis 2013;57:298-309.

7 Li H-M, Peng R-R, Li J, et al. Hiv incidence among men who have sex with men in China: a meta-analysis of published studies. PLoS One 2011;6:e23431.
8 Feng Y, Bu K, Li M, et al. [Meta-analysis of HIV infection incidence and risk factors among men who have sex with men in China]. Zhonghua Liu Xing Bing Xue Za Zhi 2015;36:752-8.

9 Arora P, Nagelkerke NJD, Jha P. A systematic review and meta-analysis of risk factors for sexual transmission of HIV in India. PLoS One 2012;7:e44094.

10 Greenwood DC. Meta-analysis of observational studies. Modern Methods for Epidemiology 2012;10:173-89.

11 Wells GA SB, O'Connell D. The Newcastle-Ottawa scale (NOS) for assessing the quality of nonrandomised studies in meta-analyses. Ottawa: Ottawa Hospital Research Institute, 2019.

12 DerSimonian R, Laird N. Meta-Analysis in clinical trials revisited. Contemp Clin Trials 2015;45:139-45.

13 Altman DG, Bland JM. Interaction revisited: the difference between two estimates. BMJ 2003;326:219.

14 Desai $\mathrm{S}$, Nardone A, Hughes $\mathrm{G}$, et al. Hiv incidence in an open national cohort of men who have sex with men attending sexually transmitted infection clinics in England. HIV Med 2017:18:615-22.

15 van Griensven F, Thienkrua W, McNicholl J, et al. Evidence of an explosive epidemic of HIV infection in a cohort of men who have sex with men in Thailand. AIDS 2013;27:825-32

16 Jin F, Prestage GP, Imrie J, et al. Anal sexually transmitted infections and risk of HIV infection in homosexual men. J Acquir Immune Defic Syndr 2010;53:144-9.

17 Ferrer L, Loureiro E, Meulbroek $M$, et al. High HIV incidence among men who have sex with men attending a community-based voluntary counselling and testing service in Barcelona, Spain: results from the ITACA cohort. Sex Transm Infect 2016;92:70-5.

18 Beymer MR, Weiss RE, Sugar CA, et al. Are centers for disease control and prevention guidelines for preexposure prophylaxis specific enough? formulation of a personalized HIV risk score for pre-exposure prophylaxis initiation. Sex Transm Dis 2017;44:49-57.

19 Beymer MR, Weiss RE, Halkitis PN, et al. Disparities within the DisparityDetermining HIV risk factors among Latino gay and bisexual men attending a community-based clinic in Los Angeles, Ca. J Acquir Immune Defic Syndr 2016;73:237-44

20 Guy RJ, Spelman T, Stoove M, et al. Risk factors for HIV seroconversion in men who have sex with men in Victoria, Australia: results from a sentinel surveillance system. Sex Health 2011:8:319-29.

21 Marcus U, Bremer V, Hamouda O, et al. Understanding recent increases in the incidence of sexually transmitted infections in men having sex with men: changes in risk behavior from risk avoidance to risk reduction. Sex Transm Dis 2006;33:11-17.

$22 \mathrm{Xu}$, An M, Han X, et al. Prospective cohort study of HIV incidence and molecular characteristics of HIV among men who have sex with men (MSM) in Yunnan Province, China. BMC Infect Dis 2013;13:3.

23 Xu J-J, Zhang M, Brown K, et al. Syphilis and HIV seroconversion among a 12-month prospective cohort of men who have sex with men in Shenyang, China. Sex Transm Dis 2010;37:432-9.

24 Yang $\mathrm{H}$, Hao $\mathrm{C}$, Huan $\mathrm{X}$, et al. Hiv incidence and associated factors in a cohort of men who have sex with men in Nanjing, China. Sex Transm Dis 2010;37:1-213.

25 Yang Z, Huang Z, Dong Z, et al. Risk factors for HIV diagnosis among men who have sex with men: results of a case-control study in one sample of eastern China. AIDS Res Hum Retroviruses 2016;32:1163-8.

26 Riedner G, Hoffmann O, Rusizoka M, et al. Decline in sexually transmitted infection prevalence and HIV incidence in female barworkers attending prevention and care services in Mbeya region, Tanzania. AIDS 2006;20:609-15.

27 Lee PM, Ho KM. Risk factors associated with human immunodeficiency virus (HIV) infection among Attendees of public sexually transmitted infection clinics in Hong Kong: implications for HIV prevention. Hong Kong Med J 2008;14:259-66.

28 Mehta SD, Ghanem KG, Rompalo AM, et al. Hiv seroconversion among public sexually transmitted disease clinic patients: analysis of risks to facilitate early identification. J Acquir Immune Defic Syndr 2006;42:116-22.

29 Ruzagira E, Wandiembe $S$, Abaasa A, et al. Hiv incidence and risk factors for acquisition in HIV discordant couples in Masaka, Uganda: an HIV vaccine preparedness study. PLoS One 2011;6:e24037.

30 Su Y, Ding G, Reilly KH, et al. Loss to follow-up and HIV incidence in female sex workers in Kaiyuan, Yunnan Province China: a nine year longitudinal study. BMC Infect Dis 2016:16:526.

31 Li D, Jia Y, Ruan Y, et al. Correlates of incident infections for HIV, syphilis, and hepatitis $B$ virus in a cohort of men who have sex with men in Beijing. AIDS Patient Care STDS 2010;24:595-602.

32 Li D, Li S, Liu Y, et al. Hiv incidence among men who have sex with men in Beijing: a prospective cohort study. BMJ Open 2012;2:001829.

33 Tang W, Babu GR, Li J, et al. The difference between HIV and syphilis prevalence and incidence cases: results from a cohort study in Nanjing, China, 2008-2010. Int J STD AIDS 2015;26:648-55.

34 Wang Q-Q, Chen X-S, Yin Y-P, et al. Hiv prevalence, incidence and risk behaviours among men who have sex with men in Yangzhou and Guangzhou, China: a cohort study. J Int AIDS Soc 2014;17:18849.

35 Braunstein SL, Ingabire CM, Kestelyn E, et al. High human immunodeficiency virus incidence in a cohort of Rwandan female sex workers. Sex Transm Dis 2011;38:385-94 
36 Solomon MM, Mayer KH, Glidden DV, et al. Syphilis predicts HIV incidence among men and transgender women who have sex with men in a preexposure prophylaxis trial. Clin Infect Dis 2014;59:1020-6.

37 Lafond RE, Lukehart SA. Biological basis for syphilis. Clin Microbiol Rev 2006;19:29-49.

38 Rekart ML, Ndifon W, Brunham RC, et al. A double-edged sword: does highly active antiretroviral therapy contribute to syphilis incidence by impairing immunity to Treponema pallidum? Sex Transm Infect 2017:93:374-8.

39 Radolf JD, Deka RK, Anand A, et al. Treponema pallidum, the syphilis spirochete: making a living as a stealth pathogen. Nat Rev Microbiol 2016;14:744-59.

40 Salazar JC, Cruz AR, Pope CD, et al. Treponema pallidum elicits innate and adaptive cellular immune responses in skin and blood during secondary syphilis: a flowcytometric analysis. J Infect Dis 2007;195:879-87.

41 Marra CM, Tantalo LC, Sahi SK, et al. Reduced Treponema pallidum-specific opsonic antibody activity in HIV-infected patients with syphilis. J Infect Dis 2016;213:1348-54.

42 Lukehart SA, Shaffer JM, Baker-Zander SA. A subpopulation of Treponema pallidum is resistant to phagocytosis: possible mechanism of persistence. J Infect Dis 1992; 166:1449-53.
43 Fitzgerald TJ. The Th1/Th2-like switch in syphilitic infection: is it detrimental? Infect Immun 1992;60:3475-9.

44 Sellati TJ, Wilkinson DA, Sheffield JS, et al. Virulent Treponema pallidum, lipoprotein, and synthetic lipopeptides induce CCR5 on human monocytes and enhance their susceptibility to infection by human immunodeficiency virus type 1. J Infect Dis 2000;181:283-93.

45 Theus SA, Harrich DA, Gaynor R, et al. Treponema pallidum, lipoproteins, and synthetic lipoprotein analogues induce human immunodeficiency virus type 1 gene expression in monocytes via NF-kappaB activation. J Infect Dis 1998;177:941-50.

46 Deng $H$, Liu R, Ellmeier W, et al. Identification of a major co-receptor for primary isolates of HIV-1. Nature 1996;381:661-6.

47 Dragic T, Litwin V, Allaway GP, et al. Hiv-1 entry into CD4+ cells is mediated by the chemokine receptor CC-CKR-5. Nature 1996;381:667-73.

48 Bentwich Z, Maartens G, Torten D, et al. Concurrent infections and HIV pathogenesis. AIDS 2000;14:2071-81.

49 World Health Organization. WHO information note on the use of dual HIV/syphilis rapid diagnostic tests (RDT), 2017. Geneva:WHO, 2019

50 Fenton KA, Breban R, Vardavas R, et al. Infectious syphilis in high-income settings in the 21st century. Lancet Infect Dis 2008:8:244-53. 